\title{
99mTc-Hydrazinonicotinamide Epidermal Growth Factor-Polyethylene Glycol-Quantum Dot Imaging Allows Quantification of Breast Cancer Epidermal Growth Factor Receptor Expression and Monitors Receptor Downregulation in Response to Cetuximab Therapy
}

Kyung-Ho Jung, Yearn Seong Choe, Jin-Young Paik, and Kyung-Han Lee

Department of Nuclear Medicine, Samsung Medical Center, Sungkyunkwan University School of Medicine, Seoul, Korea

Therapy of cancer, including basallike breast tumors, that targets the epidermal growth factor receptor (EGFR) would greatly benefit from noninvasive methods that can quantitatively monitor receptor status and treatment response. Methods: Here, we investigated the potential of a novel technique based on streptavidin cadmium selenide/zinc sulfide quantum dots (Qdots) multiplexed with polyethylene glycol (PEG), epidermal growth factor (EGF), and ${ }^{99 m T c-h y d r a z i n o n i c o t i n a m i d e . ~ I n ~ v i t r o ~}$ binding affinity and specificity were evaluated in cultured cells. Biodistribution studies and in vivo imaging were performed in murine breast tumor xenografts of basallike phenotype MDA-MB-468 cells and EGFR-negative cells. Results: ${ }^{99 m T c-}$ hydrazinonicotinamide EGF-PEG-Qdot showed specific and high-affinity EGFR targeting on confocal microscopy, immunoblotting, and binding assays. When intravenously injected, MDA-MB-468 tumors were visualized with high contrast by both optical and scintigraphic imaging. Scintigraphic image-based quantification correctly discriminated high-EGFR-expressing MDA-MB-468 tumors from other tumors, and image-based tumor uptake closely correlated to EGFR content. Importantly, serial imaging of MDA-MB-468 tumors responding to cetuximab therapy could detect a significant reduction of tumor uptake that was paralleled by downregulation of EGFR expression. Furthermore, high baseline uptake predicted good response to cetuximab therapy. Conclusion: $99 \mathrm{mTc}$-hydrazinonicotinamide EGF-PEGQdot provides EGFR-targeted imaging of breast tumors and may allow noninvasive monitoring of EGFR status in living subjects before and after targeted therapies.

Key Words: molecular imaging; oncology; breast cancer; epidermal growth factor receptor; scintigraphy

J Nucl Med 2011; 52:1457-1464

DOI: 10.2967/jnumed.111.087619

\footnotetext{
Received Jan. 7, 2011; revision accepted May 4, 2011.

For correspondence contact: Kyung-Han Lee, Department of Nuclear Medicine, Samsung Medical Center, 50 Ilwondong, Kangnamgu, Seoul, Korea.

E-mail: khnm.lee@samsung.com

Published online Aug. 17, 2011.

COPYRIGHT @ 2011 by the Society of Nuclear Medicine, Inc.
}

$\mathbf{E}$ pidermal growth factor receptors (EGFRs) play a key role in tumor growth, invasion, and metastasis (1-3) and have become a promising therapeutic target in many cancers $(4,5)$. Breast carcinoma is the most common cancer in women and a major cause of mortality, accounting for $7 \%$ of all cancer-related deaths. EGFR is overexpressed in up to $35 \%$ of breast cancers and is associated with poorer prognosis $(6,7)$. Recent data suggest potential benefits of EGFRtargeted agents not only for treating breast tumors but also for lowering their progression rate $(8)$. The efficacy of targeted therapy depends on the expression status of the molecule being aimed for, and lessons from clinical trials with EGFR antagonists have illuminated the critical importance of this factor in selecting patients most likely to respond $(4,5)$.

Molecular profiling work on breast tumors identifies a distinct and highly malignant subtype called basallike breast cancer, which is linked with higher tumor grade, more frequent metastasis, and poor clinical outcome $(9,10)$. This subgroup, with its characteristic molecular signature of negative estrogen/progesterone/HER2 receptor expression and positive EGFR expression, represents breast tumors that could most likely benefit from EGFR-targeted therapy because they lack other receptor drug targets $(9,11,12)$. The presence and level of EGFR expression is being used as an effective biomarker in many clinical studies. However, whereas limitations of conventional biopsybased methods (4) stress the need for newer image-based approaches, efforts to date have largely been unable to provide a reliable and quantitative method of monitoring tumor EGFR expression in living subjects.

In this study, we developed a novel imaging technique and investigated its ability to quantitatively monitor EGFR expression in breast tumors of basallike phenotype MDAMB-468 cells (13) and EGFR-negative MDA-MB-435 cells. The probe was based on a quantum dot (Qdot) platform surface-coated with streptavidin to which human epidermal growth factor (EGF) peptides were attached via N-terminal 
specific monoconjugation of biotinylated polyethylene glycol (PEG). Scintigraphic imaging was achieved by ${ }^{99 \mathrm{~m}} \mathrm{Tc}$-hydrazinonicotinamide conjugation to amine residues of the streptavidin. After in vitro assessment of EGFR binding characteristics, ${ }^{99 \mathrm{~m}} \mathrm{Tc}$-hydrazinonicotinamide-Qdot-PEG-EGF was investigated for its ability to assess EGFR expression in tumor-bearing mice before and after targeted therapy with cetuximab.

\section{MATERIALS AND METHODS}

\section{EGF-PEG-Qdot Synthesis and 99mTc-Hydrazinonicotinamide Labeling}

Human EGF (53 amino acids, $6.2 \mathrm{kDa}$; Cell Sciences) was conjugated to a single PEG molecule site-specifically on the Nterminal amine using slightly acidic conditions (14). This technique minimizes structural heterogeneity and influence on binding affinity associated with random conjugation. Briefly, EGF was mixed with biotin-PEG succinimidyl ester (molecular weight, 5,000; NANOCS) in $100 \mathrm{mM}$ sodium acetate (pH 5.5) at 1:1 molar ratio and incubated overnight at room temperature (RT). EGF-PEG-biotin was then attached to streptavidin-coated Qdot (655 $\mathrm{nm}$; Invitrogen) $(1 \mu \mathrm{M}$ in $30 \mu \mathrm{L})$ by 30 -min incubation at $\mathrm{RT}$ at a 90:1 molar ratio in $50 \mathrm{mM}$ sodium phosphate buffer $(\mathrm{pH}$ 8.0) with shaking. Unbound reagents were removed by a protein desalting spin column (molecular cutoff, 7,000). Qdot surface streptavidin was radiolabeled by conjugation with $20 \mu \mathrm{L}$ of hydrazinonicotinamide $(1 \mathrm{mg} / \mathrm{mL})$ at RT with shaking, followed by incubation with $370 \mathrm{MBq}$ of ${ }^{99 \mathrm{~m}} \mathrm{Tc}$ in $100 \mu \mathrm{L}$ of saline and 12 $\mu \mathrm{L}$ of tin-tricine solution at $25^{\circ} \mathrm{C}$ for $1 \mathrm{~h} .{ }^{99 \mathrm{~m}} \mathrm{Tc}-\mathrm{EGF}-\mathrm{PEG}-\mathrm{Qdot}$ was purified by a PD-10 column.

\section{Transmission Electron Microscopy}

A drop of EGF-PEG-Qdots or control Qdots in distilled water were placed on a Formvar-coated copper grid, allowed to dry for 1-2 h, and imaged by a Tecnai G2 Field Emission Transmission Electron Microscope (FEI Co.). Magnifications of 100,000- to 315,000 -fold were used, and size analysis was performed on captured digital images using Image J V. 1.34s (http://rsb.info.nih. gov/ij/).

\section{Confocal Microscopy and Cell Binding Assays}

MDA-MB-468 and MDA-MB-435 human breast cancer cells and A431 lung cancer cells were purchased from the American Type Culture Collection and maintained in $5 \% \mathrm{CO}_{2}$ at $37^{\circ} \mathrm{C}$ in Lewivovits-15 medium or high glucose Dulbecco modified Eagle medium (Lonza) supplemented with $10 \%$ fetal bovine serum, 2 $\mathrm{mM} \mathrm{L}$-glutamine, $100 \mathrm{U}$ of penicillin per milliliter, and $100 \mathrm{mg}$ of streptomycin per milliliter.

To visualize probe binding, cells on a Histobond adhesion microscope slide were fixed with $4 \%$ paraformaldehyde, incubated with EGF-PEG-Qdot or Alexa Fluor 488 EGFR antibody for $1 \mathrm{~h}$, washed, and mounted with an antifade 4',6-diamidino-2-phenylindole kit (Invitrogen). Cells were imaged with a Radiance 2100 confocal laser scanning microscope (Bio-Rad Inc.) using Lasersharp 2000 (Bio-Rad Inc.) and 543-nm excitation and 660-nm emission filters. Probe localization and EGFR staining in tumor cryosections were similarly evaluated.

For competitive binding analysis, cells were incubated with 54 $\mathrm{kBq}$ of ${ }^{99 \mathrm{~m}} \mathrm{Tc}$-EGF-PEG-Qdots (final concentration, $\sim 0.5 \mathrm{nM}$ ) for $1 \mathrm{~h}$ at $37^{\circ} \mathrm{C}$ in Dulbecco-phosphate-buffered saline and $1 \%$ bovine serum albumin. After they were washed with phosphate-buffered saline, cells were lysed with $0.5 \mathrm{~mL}$ of $0.1 \mathrm{~N} \mathrm{NaOH}$ and measured for radioactivity on a high-energy $\gamma$-counter. Binding specificity was assessed using cold EGF or an EGFR-blocking antibody. The $50 \%$ inhibitory concentration was assessed with graded concentrations of cold EGF using Prism software (version 3.02; GraphPad Software Inc.).

\section{EGFR Immunoblotting}

Extracted protein was separated by $10 \%$ sodium dodecyl sulfate polyacrylamide gel electrophoresis and transferred to a nitrocellulose membrane. After incubation with monoclonal antibodies against EGFR (rabbit) or phospho-EGFR (mouse; Tyr1068; Cell Signaling Technology, Inc.) at $4^{\circ} \mathrm{C}$ overnight, followed by incubation with secondary antibodies at RT for $1 \mathrm{~h}$, immunoreactive protein was detected with chemiluminescence and quantified using a GS800 densitometer and Quantity One software (Bio-Rad Laboratories).

\section{In Vivo Pharmacokinetics and Biodistribution Studies}

All animal experiments were in accordance with the National Institutes of Health Guide for the Care and Use of Laboratory Animals (15) and approved by the institutional committee. Tumor models were prepared in BALB/c nude mice by subcutaneous injection of $1 \times 10^{8}$ cells into the right shoulder. MDA-MB-468 cell xenografts were used as a model for high-EGFR-expressing basallike breast cancers. Xenografts of mixed MDA-MB-468 and MDA-MB-435 cells (3:1 ratio) and MDA-MB-435 cells were used as intermediate- and low-EGFR-expressing breast tumor models, respectively. Experiments were performed when tumor diameter reached approximately $1.0 \mathrm{~cm}$.

Pharmacokinetic analysis was performed in normal imprinting control region (ICR) mice intravenously injected with $1.1 \mathrm{MBq}$ of ${ }^{99 m}$ Tc-EGF-PEG-Qdots. Blood collected from the tail vein was measured for radioactivity, and parameters of half-life, clearance, and volume of distribution were calculated. Early distribution and late clearance rate constants $\left(K_{1}\right.$ and $\left.K_{2}\right)$ and half-lives (T1/2 $\alpha$ and $\mathrm{T} 1 / 2 \beta$ ) were derived by nonlinear regression fitting of the timeactivity curve with a 2-phase exponential decay equation using Prism software (version 3.02). Biodistribution studies were performed in MDA-MB-468 tumor-bearing mice intravenously injected with 18.5 MBq of ${ }^{99 m} \mathrm{Tc}$-EGF-PEG-Qdots. Major organs and tumor were extracted at $4 \mathrm{~h}$, weighed, and measured for radioactivity.

\section{Cetuximab Therapy and Tumor Response Measurement}

Tumor-bearing mice were treated with intraperitoneal administration of cetuximab (Merck Korea) at a dose of $1 \mathrm{mg}$ per mouse or saline (controls) on days 0,3 , and 6 (16). Tumors were measured daily by a caliper and calculated for tumor volume with the widely used modified ellipsoidal formula (17): tumor volume $\left(\mathrm{mm}^{3}\right)=$ length $\times$ width $^{2} \times 1 / 2$. Change in tumor size was expressed as percentage volume, compared with that at day 0 .

\section{In Vivo Optical and Scintigraphic Imaging}

In vivo fluorescence imaging of mice was performed with an IVIS Spectrum imaging system (Xenogen; 605-nm excitation, 660-nm emission) at $4 \mathrm{~h}$ after injection of $10 \mu \mathrm{M}$ EGF-PEG-Qdot in a volume of $200 \mu \mathrm{L}$. Ex vivo optical images of tumors and major organs were obtained after extraction from sacrificed animals. 
Scintigraphic imaging was performed in tumor-bearing mice the day before initiating cetuximab therapy (baseline) and again at $3 \mathrm{~d}$ after the last treatment dose (after therapy). Animals were injected via the tail vein with $18.5 \mathrm{MBq}$ of ${ }^{99 m}$ Tc-EGF-PEGQdots and imaged $4 \mathrm{~h}$ later under isoflurane anesthesia. Imaging at $4 \mathrm{~h}$ after injection was chosen because preliminary experiments between 1 and $4 \mathrm{~h}$ showed the best tumor contrast at this time. Imaging was performed on a $\gamma$-camera (Trionix Research Laboratory) with an intrinsic spatial resolution of less than $3.8 \mathrm{~mm}$ in full width at half maximum, equipped with a 2-mm aperture pinhole collimator. A $15 \%$ energy window centered around $140 \mathrm{keV}$ was used, and 15-min image data were stored on a $256 \times 256$ pixel matrix. Regions of interest were drawn on the tumor and on the contralateral limb to obtain image-based tumor-to-background (T/B) ratios of activity.

\section{Statistical Analysis}

Data are presented as mean \pm SD unless otherwise specified. Significance of difference between multiple groups was determined by 1-way ANOVA with post hoc Bonferroni multiple comparison tests. Correlation was evaluated by linear regression analysis.

\section{RESULTS}

\section{EGF-PEG-Qdot Preparation and In Vitro EGFR Targeting}

Preparation of ${ }^{99 m}$ Tc-EGF-PEG-Qdots (Fig. 1A) was straightforward and reproducible. Biotin-PEG served both as linker for Qdot attachment and flexible spacer to allow receptor binding without steric hindrance. Radiolabeling with ${ }^{99 m}$ Tc-hydrazinonicotinamide was achieved with high radiochemical yield and specific activity. The Qdots are typically surface-conjugated with 5-10 streptavidins, which are tetramers with a biotin binding site on each subunit. Because only a portion of these sites is expected to be available for biotin binding, there may be somewhere around 10 EGF-PEG molecules attached to each Qdot. Similarly, a comparable number of hydrazinonicotinamide molecules, which attach to lysine residues present in streptavidin, may be attached to each Qdot. Transmission electron microscopy displayed approximately 15-nm particles without clustering (Fig. 1A).

Treatment of MDA-MB-468 cells with 5 nM EGF-PEGQdot for 2 min caused phosphorylation of cellular EGFR, with potency comparable to that of free EGF peptides (Fig. 1B). This property should be beneficial for imaging because internalization of the activated ligand-receptor complex can facilitate probe accumulation.

Confocal microscopy visualized direct binding of EGFPEG-Qdots to MDA-MB-468 cell surface, in a fashion similar to fluorescent anti-EGFR antibodies (Fig. 2A). Cell binding analysis confirmed receptor specificity of probe binding that was reduced $58.1 \% \pm 2.4 \%$ by $100 \mathrm{nM}$ cold EGF and $64.5 \% \pm 1.7 \%$ by $100 \mathrm{nM}$ anti-EGFR-blocking antibody in MDA-MB-468 cells, and $78.9 \% \pm 1.4 \%$ and $85.7 \% \pm 1.7 \%$, respectively, in A431 lung cancer cells (Fig. 2B). Competitive binding assays showed high-affinity binding to both MDA-MB-468 and A431 cells (Fig. 2C).
These results confirm that EGF attached via N-terminal monoconjugated PEG to ${ }^{99 m}$ Tc-hydrazinonicotinamidelabeled Qdots retains specific and high-affinity binding to functional EGFR.

\section{Pharmacokinetics and In Vivo Tumor Targeting of EGF-PEG-Qdots}

Intravenously injected ${ }^{99 \mathrm{~m} T c-E G F-P E G-Q d o t s ~ i n ~ n o r m a l ~}$ mice displayed a biexponential profile of blood clearance with early distribution $\left(K_{1}\right)$ and late clearance rate constants $\left(K_{2}\right)$ of 0.598 and $0.026 \mathrm{~min}^{-1}$, respectively (Fig. 3A, left). Circulation $\mathrm{T} 1 / 2 \alpha$ was $1.2 \mathrm{~min}$ and $\mathrm{T} 1 / 2 \beta$ was $26.6 \mathrm{~min}$, the volume of distribution was $31.46 \mathrm{~mL}$, and clearance was $1.18 \mathrm{~mL} / \mathrm{min}$.

Biodistribution of $99 \mathrm{~m}$ Tc-EGF-PEG-Qdot in MDA-MB468 tumor-bearing nude mice at $4 \mathrm{~h}$ revealed high tumor accumulation that reached $3.41 \% \pm 0.15 \%$ injected dose per gram of tissue (\%ID/g; Fig. 3A, right). This was a level 4.6-fold greater than blood and 9.8-fold greater than muscle activity. Remarkably, tumor uptake exceeded even that of liver tissue $(2.96 \pm 0.07 \% \mathrm{ID} / \mathrm{g})$ —an unexpected finding given the prominent reticuloendothelial system phagocytosis and uptake of most Qdot particles. Specificity of tumor targeting was demonstrated by inhibition experiments with preinjection of EGFR-blocking antibody, which caused a $59.3 \% \pm 0.7 \%$ reduction in tumor uptake $(P<0.005$; Fig. 3A, right). Stomach and thyroid activity was low (not shown), indicating that there is no significant in vivo release of free ${ }^{99 \mathrm{~m}} \mathrm{Tc}$ from the probe.

In vivo optical imaging of MDA-MB-468 tumor-bearing mice $4 \mathrm{~h}$ after EGF-PEG-Qdot injection displayed clear visualization of tumor nodules, with strong fluorescent signals (Fig. 3B, left). This finding was corroborated by ex vivo optical imaging of extracted organ and tumor tissues (Fig. 3B, right). The tumor tissue was then microsectioned and subjected to confocal microscopic inspection, revealing that the microscopic distribution of injected Qdots in tumor corresponded to tissues expressing EGFR (Fig. 3C). Scintigraphic acquisition at $4 \mathrm{~h}$ after injection also displayed high-contrast tumor ${ }^{99 \mathrm{~m}}$ Tc-EGF-PEG-Qdot uptake (Fig. 3D). Taken together, these results provide evidence for active in vivo tumor EGFR targeting of our probe.

\section{Tumor Images Before and After Cetuximab Treatment}

On baseline ${ }^{99 m}$ Tc-EGF-PEG-Qdot images, T/B ratios were significantly greater in high-EGFR MDA-MB-468 tumors than in other tumors $(2.19 \pm 0.50$ vs. $1.50 \pm$ $0.33 ; P=0.002$ ). Hence, a $\mathrm{T} / \mathrm{B}$ ratio of 1.85 had $90 \%$ sensitivity and specificity for identifying tumors with high-EGFR expression (Fig. 4A, left).

After therapy, only the cetuximab-treated MDA-MB-468 group, compared with saline-injected MDA-MB-468 controls, showed retardation of tumor growth (Fig. 4A, right). Quantitative analysis of ${ }^{99 m}$ Tc-EGF-PEG-Qdot images in control MDA-MB-468 group animals showed a $49.4 \%$ 
A

FIGURE 1. Probe characterization. (A) Scheme of $99 \mathrm{mTc}$-hydrazinonicotinamide EGF-PEG-Qdots (left) and transmission electron microscopy findings (right). Probe is composed of streptavidin-coated Qdot platform multiplexed with PEG-biotin monoconjugated EGF and 99mTc-hydrazinonicotinamide. Transmission electron microscopy shows similar 10- to15-nm size and morphology of control Qdots (top) and EGFPEG-Qdots (bottom). (B) Immunoblots of MDA-MB-468 cells show increased phosphorylation of EGFR by stimulation with 5 nM human EGF or EGF-PEG-Qdot but not control PEG-Qdot. HYNIC = hydrazinonicotinamide; Sav $=$ streptavidin.

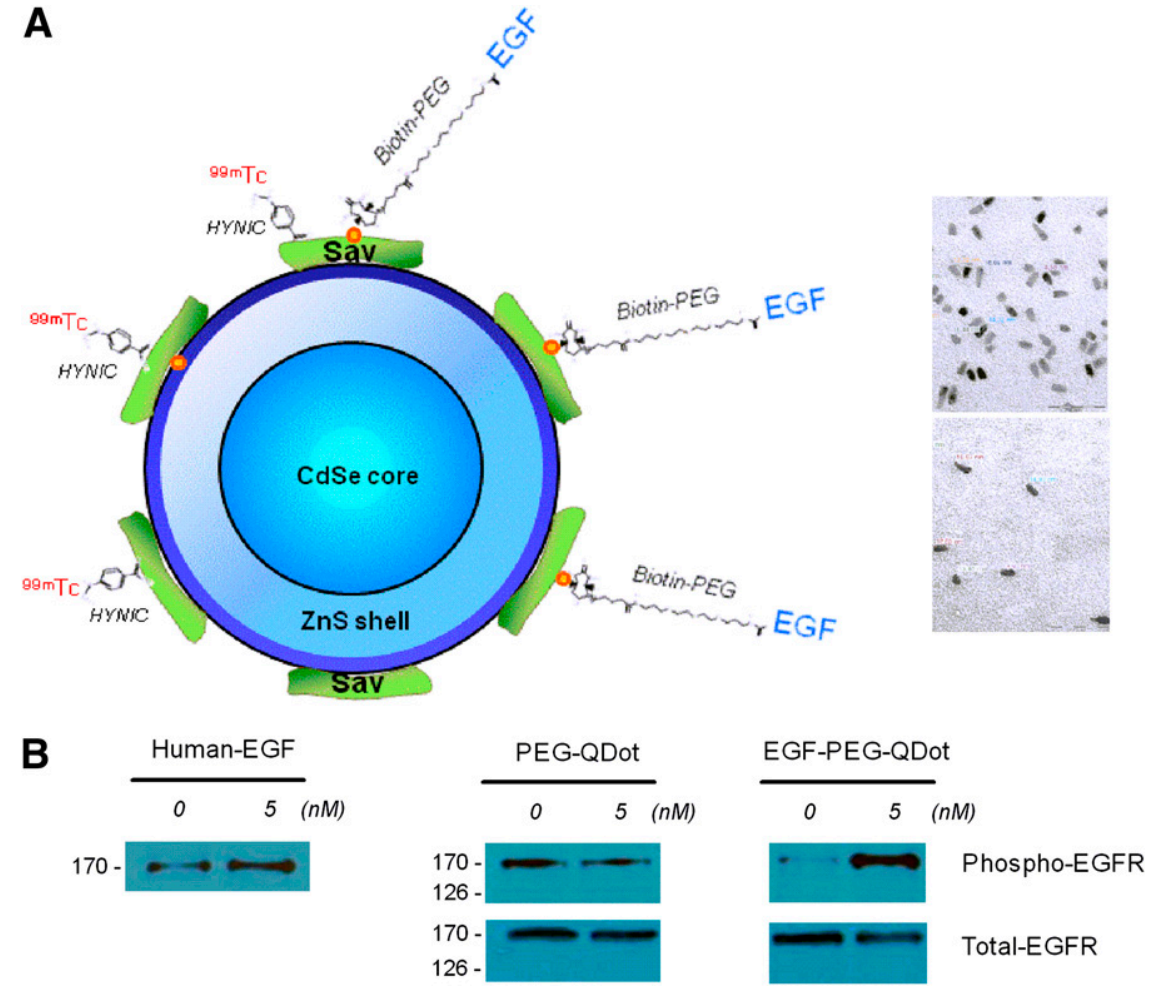

increase of $\mathrm{T} / \mathrm{B}$ ratio from $2.21 \pm 0.31$ to $2.81 \pm 0.55$ between baseline and follow-up scintigraphic studies. In contrast, MDA-MB-468 tumor-bearing mice treated with cetuximab displayed a significant $(25.3 \%)$ reduction of T/B ratio from $2.09 \pm 0.58$ to $1.83 \pm 0.28$ during the same time (Fig. 4B, left; Supplemental Fig. 1 [supplemental materials are available online only at http://jnm.snmjournals.org]). Cetuximab treatment-induced reduction in tumor uptake
FIGURE 2. In vitro EGFR binding. (A) Confocal microscope of $4^{\prime}, 6$-diamidino-2-phenylindole-stained (blue) MDA-MB-468 cells show cell surface binding of fluorescent anti-EGFR antibodies (green; top) and EGF-PEG-Qdots (red; bottom). (B) Specific binding of $99 \mathrm{mT}$ T-hydrazinonicotinamide EGF-PEG-Qdots to MDA-MB-468 and A431 cells was demonstrated by excess cold EGF or EGFR-blocking antibody (Ab). (C) Competitive binding of $99 \mathrm{mTc}$-hydrazinonicotinamide EGF-PEG-Qdot with graded concentrations of cold EGF in MDA-MB468 (left) and A431 cells (right). All results are mean $\pm S D$ from single representative experiment of 2 experiments done in triplicate. ${ }^{* \star *} P<0.001$, compared with uninhibited controls by 1-way ANOVA. $\mathrm{IC}_{50}=$ $50 \%$ inhibitory concentration.

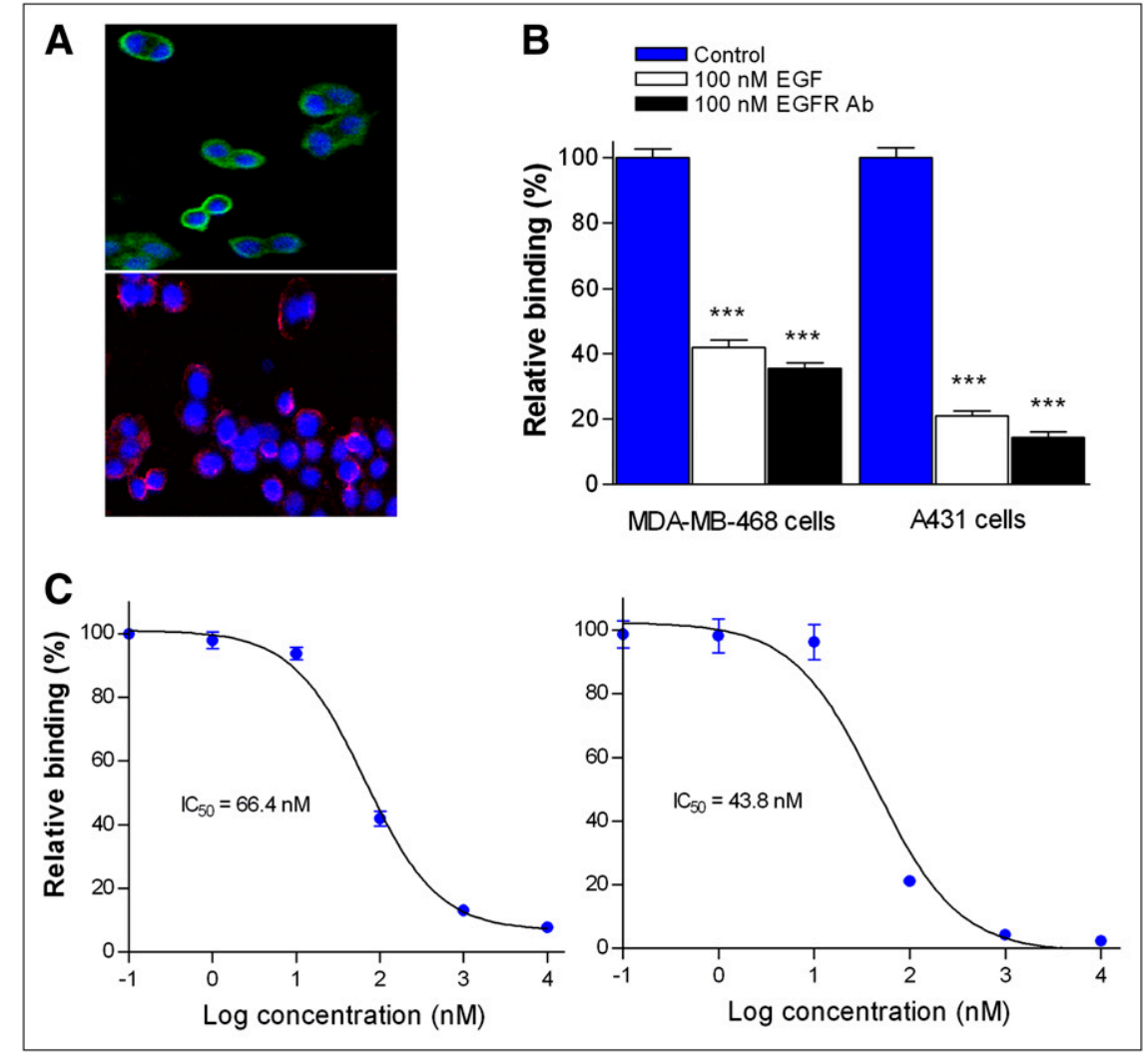




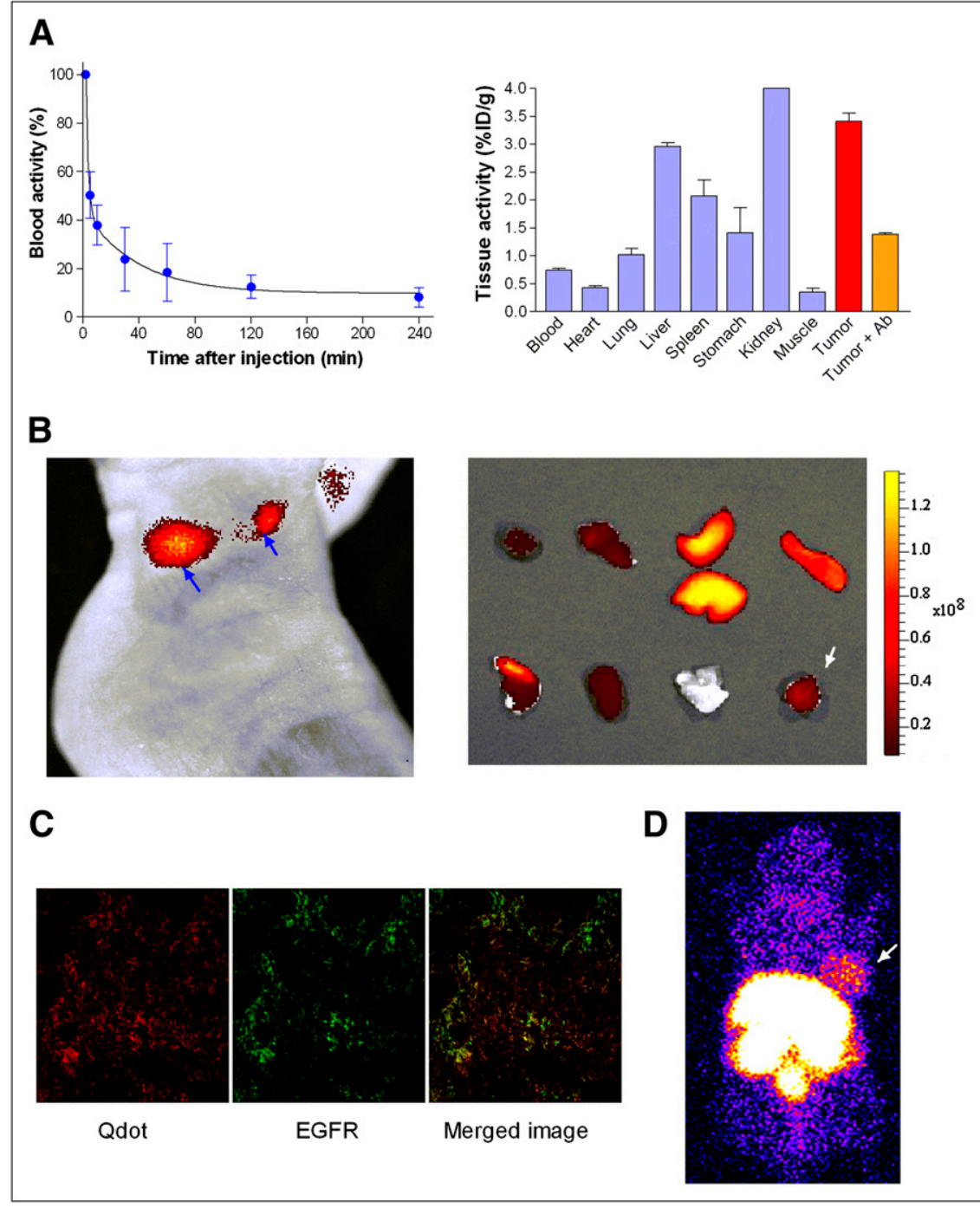

FIGURE 3. Enhanced in vivo kinetics and tumor-targeted imaging. (A) Blood activity kinetics (left) and biodistribution at $4 \mathrm{~h}$ (right) after ${ }^{99 m T c}$-hydrazinonicotinamide EGFPEG-Qdot injection. Blood activity data are mean \pm SD of percentage initial activity in 5 normal ICR mice. Biodistribution data are mean \pm SD of 2 MBA-MB-468 tumor mice with 2 separate animals for blocking data, and results show high tumor uptake that was inhibited by blocking antibody (Tumor $+A b)$. (B) In vivo fluorescent image at $4 \mathrm{~h}$ (left) and ex vivo fluorescent image of tissues of same animal (right). In vivo EGF-PEG-Qdot accumulation is seen in 2 MDA-MB-468 tumor nodules (arrows). Ex vivo tissues from top left to bottom right are heart, lung, liver, spleen, stomach, kidney, muscle, and tumor (arrow). (C) Confocal microscope of cryosection from tumor in B shows EGF-PEG-Qdots (left), fluorescent antibody-stained EGFR (middle), and colocalization of 2 signals on merging (right). Magnification, $\times 400$. (D) Scintigraphic images $4 \mathrm{~h}$ after $99 \mathrm{mTc}$-hydrazinonicotinamide EGF-PEG-Qdot injection displaying high-contrast MDA-MB-468 tumor uptake (arrow). was confirmed by ex vivo measurements, demonstrating a $47.2 \%$ lower uptake level for cetuximab-treated MDA-MB468 tumors than for saline-treated controls $(0.91 \pm 0.18$ vs. $1.73 \pm 0.39 \% \mathrm{ID} / \mathrm{g}$; Fig. 4B, right).

\section{Image Results Correlate to EGFR Expression and Cetuximab Response}

Immunoblotting of EGFR levels of posttherapy tumor tissue displayed findings consistent with results of scintigraphic quantification. Thus, EGFR expression was highest in saline-injected MDA-MB-468 tumors, followed by significantly lower expression in cetuximab-treated MDAMB-468 and MDA-MB-468/435 tumors (Supplemental Fig. 2). EGFR-negative MDA-MB-435 tumors showed undetectable levels.

In assessing the correlation of $99 \mathrm{~m}$ Tc-EGF-PEG-Qdot uptake to EGFR amount, we first confirmed that imagebased T/B ratio closely correlates to ex vivo-measured tumor uptake ( $r=0.89, P=0.001$; data not shown). We then showed that both image-based T/B ratio and ex vivo tumor uptake correlate well to immunoblot-measured EGFR amount (Fig. 5A). These results indicate that 99m Tc-EGFPEG-Qdot imaging can be a reliable technique for noninvasive assessment of tumor EGFR status.

Finally, we observed a significant inverse correlation between baseline T/B ratio and tumor growth rate during cetuximab therapy (Fig. 5B, left). Receiver-operator-characteristic curve analysis showed that a threshold baseline T/B ratio of 1.7 could discriminate tumors with greater growth inhibition by cetuximab with a sensitivity of $100 \%$ and specificity of $75 \%$ (area under curve, 0.8; Fig. 5B, right).

\section{DISCUSSION}

In this study, we demonstrate that quantitative scintigraphic assessment of breast tumor EGFR expression is both feasible and reliable using a ${ }^{99 \mathrm{~m}}$ Tc-hydrazinonicotinamide-labeled mono-PEG-EGF-Qdot probe with high-affinity receptor binding and favorable in vivo characteristics. There was close correlation between image-based tumor uptake and actual EGFR content. Importantly, basallike phenotype MDA-MB-468 breast tumors revealed a significant reduc- 
FIGURE 4. Effects of cetuximab therapy in tumor mice. (A) Baseline image-based T/B count ratios (left) and serial tumor volume in tumor groups (right). Baseline T/B ratios effectively discerned high-EGFR-expressing from low-EGFR-expressing tumors. Dotted line depicts differentiating threshold T/B ratio of 1.85 . Serial tumor volumes are mean \pm SE of percentage tumor volume in cetuximab- or saline-treated groups relative to that at day $0 . n=5$ for each group, except for MDA-MB-468-saline group in which 1 animal died during follow-up. ${ }^{\star} P<$ 0.05 between MDA-MB-468-ctx and MDAMB-435-ctx groups. (B) Image-based T/B ratios (left) and ex vivo-measured tumor uptake (right) after treatment. Bars represent mean \pm SD of 4 or 5 tumors per group. ${ }^{\star \star} P<0.01 ;{ }^{\star \star \star} P<0.005$, compared with MDA-MB-468-saline group by 1-way ANOVA. ctx $=$ cetuximab.
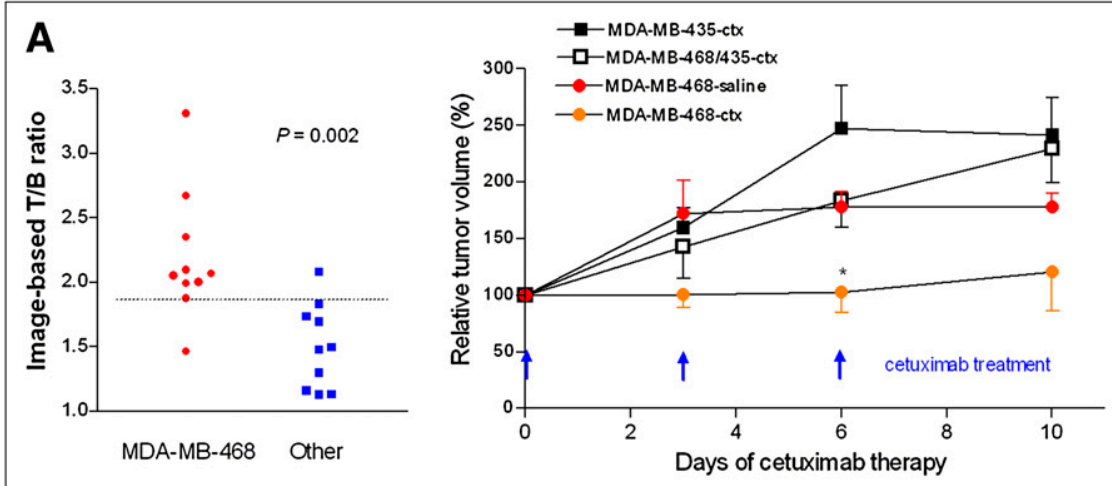

RGB
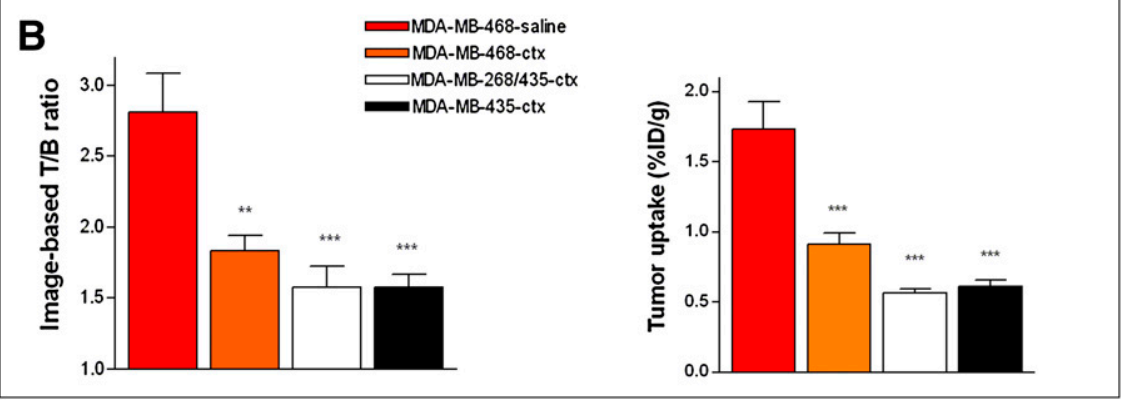

Molecular imaging of the global status of drug targets in living subjects could help select the right subset of patients to demonstrate the drug's true potential and limit exposure to therapies unlikely to be beneficial $(18,19)$. Breast cancers tion of ${ }^{99 m}$ Tc-hydrazinonicotinamide PEG-EGF-Qdot uptake by cetuximab therapy that was paralleled by EGFR downregulation. Furthermore, baseline image results correlated with magnitudes of response to targeted treatment.

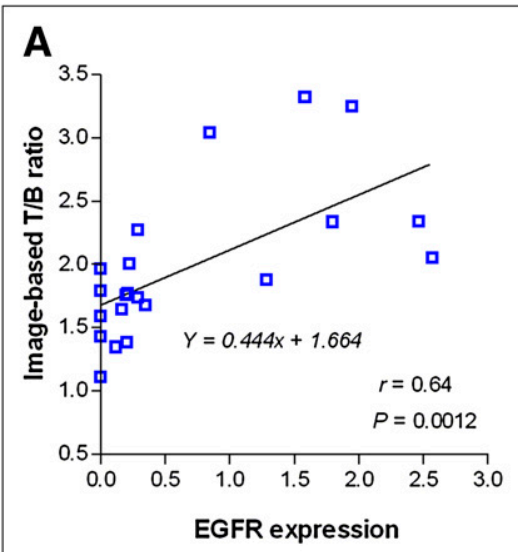

B

FIGURE 5. Tumor uptake vs. EGFR expression and therapy response. (A) Correlation between $T / B$ ratio (left) or ex vivo tumor uptake (right) and tumor EGFR content. (B) Correlation between $T / B$ ratio and cetuximab response. There was significant inverse correlation between baseline T/B ratio and cetuximab response (percentage increase of tumor volume; left). Receiveroperator-characteristic curve shows high performance of high T/B ratios $(>1.7)$ for predicting cetuximab response $(<90 \%$ tumor volume increase; right). Correlations are assessed by linear-regression analysis.

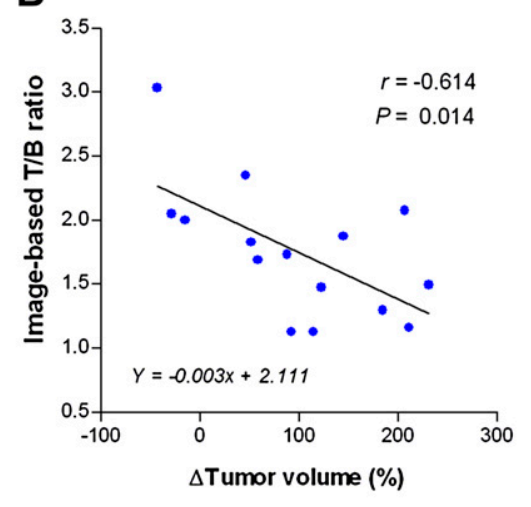


that are negative for receptors for estrogen, progesterone, and $H E R 2$ respond unfavorably to conventional therapy, partly because of growth stimulation from overexpressed EGFR. Thus, EGFR targeting might have significant therapeutic and preventive potential for malignancies of the breast, and preliminary clinical studies have shown moderate clinical efficacy $(8,12)$. Hence, the development of new imaging biomarkers could help advance our understanding of the mechanism of anti-EGFR therapy in breast cancer and provide longitudinal monitoring of response.

The present study demonstrates that scintigraphic imaging with EGF-labeled nanoprobes may be a useful strategy for monitoring breast tumor EGFR expression and response to cetuximab treatment. In our methods, human EGF was used as targeting ligand because peptide probes are the most suited for selective tracing of functionally active high-affinity surface receptors. Compared with antibodies, they have greater binding affinity, better tumor penetration, and more rapid blood clearance, which together result in improved T/B ratios. On the other hand, peptides are susceptible to loss of binding affinity during conjugation, have low in vivo metabolic stability, and are often cleared from the blood at excessive rates $(20,21)$. We overcame these difficulties using a nanoparticle platform to which EGF was conjugated in a manner that minimized influence on structural and functional integrity. The use of biotinPEG as linker to streptavidin is likely to have further enhanced the in vivo stability of EGF and may also have contributed to an improvement of in vivo kinetics of the probe.

As a platform to integrate PEG-EGF and ${ }^{99 \mathrm{~m}} \mathrm{Tc}$, we used Qdots, a new class of semiconductor optical reporters that provide a versatile nanoscale scaffold for multifunctional targeted imaging or therapy (22-24). Single-photon labeling with ${ }^{99 \mathrm{~m}}$ Tc allowed an imaging time window that matched well the pharmacokinetics of peptide ligands and provided sensitive detection of probe distribution. Because scintigraphic images are attained by injection of small numbers of probe molecules, ${ }^{99 \mathrm{~m} T c}$ labeling also circumvented concern for toxicity issues that depend on administered Qdot dose (25).

Another difficulty for in vivo Qdot imaging is prominent hepatosplenic accumulation $(22,26-28)$ that not only reduces target binding opportunity but also degrades overall image quality. A notable favorable kinetic property of our technique was relatively low hepatosplenic activity that contributed to high-quality tumor images. Although several factors can influence Qdot clearance, the low liver uptake of our probe could be related to passivating effects of surface PEG or hydrazinonicotinamide (29). In any case, superior binding and in vivo kinetic properties of our probe provided high-contrast tumor images that compare favorably to previous EGF-conjugated fluorophore (30-32) or Qdot (33) probes. However, scintigraphic imaging with single photon-emitting radiopharmaceuticals, compared with PET techniques, has several limitations, including lower sensitivity, resolution, and quantitative capacity.
Evidence that ${ }^{99} \mathrm{~m}$ Tc-hydrazinonicotinamide PEG-EGFQdot images actually reflect in vivo receptor targeting was provided by localization of injected probes to regions positive for EGFR staining, inhibition of tumor uptake by EGFR-blocking antibodies, and close correlation between magnitudes of tumor uptake and EGFR content. Although immunohistochemistry is the current clinic standard for assessing receptor status, it is associated with many technical shortcomings including disparity between receptor epitope targeted by immunostaining and therapeutic drugs, sampling error, poor discrimination between low- and high-affinity receptors, and tissue-processing issues. Immunohistochemical scoring systems also lack standardization and are highly subject to interobserver variation (4). In our study, we quantified global tumor EGFR content by measuring immune reactive band intensities from protein of whole tumor tissue. As a result, both image-based T/B ratios and ex vivo measurements of tumor uptake were shown to closely correlate to EGFR expression.

Importantly, our imaging technique was able to detect alterations in EGFR expression in response to targeted therapy with cetuximab, the most commonly used anti-EGFR agent for various solid tumors including breast cancer. When we treated tumor-bearing mice with cetuximab, high-EGFRexpressing MDA-MB-468 tumors showed a significant reduction of ${ }^{99 m}$ Tc-PEG-EGF-Qdot uptake that was paralleled by a decrease in EGFR expression. This finding is consistent with recent reports that antibodies against EGFR not only block ligand binding but also can induce receptor internalization and lysosomal degradation, leading to EGFR downregulation (34,35). Finally, tumor ${ }^{99 \mathrm{~m} T c-P E G-E G F-Q d o t}$ uptake on baseline images correlated inversely to the extent of tumor growth after cetuximab therapy.

\section{CONCLUSION}

99m Tc-PEG-EGF-Qdot provides high-contrast imaging of breast tumor xenografts via specific and efficient in vivo receptor targeting. Scintigraphic imaging offers noninvasive quantification of tumor EGFR content and can detect EGFR downregulation by cetuximab treatment in responsive breast tumors. These results demonstrate the encouraging potential of this imaging strategy for the noninvasive monitoring of EGFR status in living subjects before and after targeted therapies.

\section{DISCLOSURE STATEMENT}

The costs of publication of this article were defrayed in part by the payment of page charges. Therefore, and solely to indicate this fact, this article is hereby marked "advertisement" in accordance with 18 USC section 1734.

\section{ACKNOWLEDGMENTS}

This work was supported by a grant from the National R\&D Program for Cancer Control, Ministry of Health \& 
Welfare, Republic of Korea (grant number 0720190-2). No other potential conflict of interest relevant to this article was reported.

\section{REFERENCES}

1. Yarden Y, Sliwkowski MX. Untangling the ErbB signalling network. Nat Rev Mol Cell Biol. 2001;2:127-137.

2. Arteaga CL. The epidermal growth factor receptor: from mutant oncogene in nonhuman cancers to therapeutic target in human neoplasia. J Clin Oncol. 2001;19(18 suppl):32S-40S.

3. Sainsbury JR, Farndon JR, Needham GK, Malcolm AJ, Harris AL. Epidermalgrowth-factor receptor status as predictor of early recurrence of and death from breast cancer. Lancet. 1987;1:1398-1402.

4. Siena S, Sartore-Bianchi A, Di Nicolantonio F, Balfour J, Bardelli A. Biomarkers predicting clinical outcome of epidermal growth factor receptor-targeted therapy in metastatic colorectal cancer. J Natl Cancer Inst. 2009;101:1308-1324.

5. Baselga J, Arteaga CL. Critical update and emerging trends in epidermal growth factor receptor targeting in cancer. J Clin Oncol. 2005;23:2445-2459.

6. Bossuyt V, Fadare O, Martel M, et al. Remarkably high frequency of EGFR expression in breast carcinomas with squamous differentiation. Int J Surg Pathol. 2005;13:319-327.

7. Pawlowski V, Révillion F, Hebbar M, Hornez L, Peyrat JP. Prognostic value of the type I growth factor receptors in a large series of human primary breast cancers quantified with a real-time reverse transcription-polymerase chain reaction assay. Clin Cancer Res. 2000;6:4217-4225.

8. Gao XP, Liu F. New agents in development for breast cancer. Curr Opin Obstet Gynecol. 2007;19:68-74.

9. Sorlie T, Perou CM, Tibshirani R, et al. Gene expression patterns of breast carcinomas distinguish tumor subclasses with clinical implications. Proc Natl Acad Sci USA. 2001;98:10869-10874.

10. Da Silva L, Clarke C, Lakhani SR. Demystifying basal-like breast carcinomas. $J$ Clin Pathol. 2007;60:1328-1332.

11. Livasy CA, Karaca G, Nanda R, et al. Phenotypic evaluation of the basal-like subtype of invasive breast carcinoma. Mod Pathol. 2006;19:264-271.

12. Flynn JF, Wong C, Wu JM. Anti-EGFR therapy, mechanism and advances in clinical efficacy in breast cancer. J Oncol. 2009;2009:526963.

13. Neve RM, Chin K, Fridlyand J, et al. A collection of breast cancer cell lines for the study of functionally distinct cancer subtypes. Cancer Cell. 2006;10:515527.

14. Lee H, Jang IH, Ryu SH, Park TG. N-terminal site-specific mono-PEGylation of epidermal growth factor. Pharm Res. 2003;20:818-825.

15. Guide for the Care and Use of Laboratory Animals. Bethesda, MD: National Institutes of Health; 1985. NIH publication 85-23.

16. Rose WC, Wild R. Therapeutic synergy of oral taxane BMS-275183 and cetuximab versus human tumor xenografts. Clin Cancer Res. 2004;10:7413-7417.

17. Euhus DM, Hudd C, LaRegina MC, Johnson FE. Tumor measurement in the nude mouse. J Surg Oncol. 1986;31:229-234.
18. Weissleder R, Pittet MJ. Imaging in the era of molecular oncology. Nature. 2008;452:580-589.

19. Mankoff DA, Sullivan FO, Barlow WE, Krohn KA. Molecular imaging research in the outcomes era: measuring outcomes for individualized cancer therapy. Acad Radiol. 2007;14:398-405.

20. Maecke HR. Radiolabeled peptides in nuclear oncology: influence of peptide structure and labeling strategy on pharmacology. Ernst Schering Res Found Workshop. 2005;49:43-72.

21. Liu S. Bifunctional coupling agents for radiolabeling of biomolecules and targetspecific delivery of metallic radionuclides. Adv Drug Deliv Rev. 2008;60:13471370 .

22. Michalet X, Pinaud FF, Bentolila LA, et al. Quantum dots for live cells, in vivo imaging, and diagnostics. Science. 2005;307:538-544.

23. Gao X, Cui Y, Levenson RM, Chung LW, Nie S. In vivo cancer targeting and imaging with semiconductor quantum dots. Nat Biotechnol. 2004;22:969976.

24. Tada $\mathrm{H}$, Higuchi $\mathrm{H}$, Wanatabe TM, Ohuchi N. In vivo real-time tracking of single quantum dots conjugated with monoclonal anti-HER2 antibody in tumors of mice. Cancer Res. 2007;67:1138-1144.

25. Hardman R. A toxicologic review of quantum dots: toxicity depends on physicochemical and environmental factors. Environ Health Perspect. 2006;114:165172.

26. Schipper ML, Cheng Z, Lee SW, et al. MicroPET-based biodistribution of quantum dots in living mice. $J$ Nucl Med. 2007;48:1511-1518.

27. Cai W, Shin DW, Chen K, et al. Peptide-labeled near-infrared quantum dots for imaging tumor vasculature in living subjects. Nano Lett. 2006;6:669-676.

28. Owens DE 3rd, Peppas NA. Opsonization, biodistribution, and pharmacokinetics of polymeric nanoparticles. Int J Pharm. 2006;307:93-102.

29. Schipper ML, Iyer G, Koh AL, et al. Particle size, surface coating, and PEGylation influence the biodistribution of quantum dots in living mice. Small. 2009;5:126-134.

30. Manning HC, Merchant NB, Foutch AC, et al. Molecular imaging of therapeutic response to epidermal growth factor receptor blockade in colorectal cancer. Clin Cancer Res. 2008;14:7413-7422.

31. Wang K, Wang K, Li W, et al. characterizing breast cancer xenograft epidermal growth factor receptor expression by using near-infrared optical imaging. Acta Radiol. 2009;50:1095-1103.

32. Ke S, Wen X, Gurfinkel M, et al. Near-infrared optical imaging of epidermal growth factor receptor in breast cancer xenografts. Cancer Res. 2003;63:78707875 .

33. Diagaradjane P, Orenstein-Cardona JM, Colón-Casasnovas NE, et al. Imaging epidermal growth factor receptor expression in vivo: pharmacokinetic and biodistribution characterization of a bioconjugated quantum dot nanoprobe. Clin Cancer Res. 2008;14:731-741.

34. Perez-Torres M, Guix M, Gonzalez A, Arteaga CL. Epidermal growth factor receptor (EGFR) antibody down-regulates mutant receptors and inhibits tumors expressing EGFR mutations. J Biol Chem. 2006;281:40183-40192.

35. Oliveira S, Schiffelers RM, van der Veeken J, et al. Downregulation of EGFR by a novel multivalent nanobody-liposome platform. J Control Release. 2010;145: $165-175$. 